\title{
Ultralow Threshold Optical Gain Enabled by Quantum Rings of Inverted Type-I CdS/CdSe Core/Crown Nanoplatelets in the Blue
}

\author{
Savas Delikanli, Furkan Isik, Farzan Shabani, Hamed Dehghanpour Baruj, \\ Nima Taghipour, and Hilmi Volkan Demir'*
}

\begin{abstract}
Achieving low-threshold optical gain for solution-processed materials is crucial for their real-life applications and deployment as gain media. However, the realization of low gain threshold in the blue region has shown to be technically an extremely challenging task using colloidal nanocrystals as a result of fast nonradiative Auger rates in smaller nanocrystals. Here, ultralow-threshold blue amplified spontaneous emission (ASE) $\left(\approx 2.7 \mu \mathrm{cm}^{-2}\right)$ accompanied with a large net modal gain coefficient of $360 \mathrm{~cm}^{-1}$ in the blue enabled by blue-emitting ( $\approx 455-465 \mathrm{~nm}$ ) colloidal quantum rings (QRs) of inverted type-I CdS/CdSe core/crown nanoplatelets (NPLs) is proposed and demonstrated. The synthesized QRs with controlled crown size outperform the best reported ASE thresholds and net modal gain coefficients from the solution-processed materials by $\approx 2.5$ - and $\approx 4$-fold, respectively, in the similar blue spectral window. Utilizing this QR architecture, it is also shown that the ASE peak can be spectrally tuned by controlling the lateral size of the crown and hence quantum confinement in the lateral direction. These outstanding results support the prospects of these solution-processed QRs made of 2D hetero-NPLs in the challenging blue region as colloidal gain.
\end{abstract}

\section{Introduction}

2D semiconductor nanoplatelets (NPLs), which make colloidal quantum wells, recently have emerged as a favorable and novel platform for optoelectronic applications, including

Dr. S. Delikanli, Prof. H. V. Demir

Luminous! Center of Excellence for Semiconductor Lighting and Displays

School of Electrical and Electronic Engineering

School of Physical and Mathematical Sciences

Nanyang Technological University

Singapore 639798, Singapore

E-mail:volkan@bilkent.edu.tr

Dr. S. Delikanli, F. Isik, F. Shabani, H. D. Baruj, N. Taghipour,

Prof. H. V. Demir

Department of Electrical and Electronics Engineering

Department of Physics

UNAM - Institute of Materials Science and Nanotechnology

Bilkent University

Ankara 06800, Turkey

The ORCID identification number(s) for the author(s) of this article can be found under https://doi.org/10.1002/adom.202002220.

DOI: 10.1002/adom.202002220 light-emitting diodes and lasing, ${ }^{[1]}$ as a result of their unique optical and electronic properties such as giant oscillator strength, ${ }^{[2]}$ exceptional color purity owing to their magic-size vertical thickness, ${ }^{[3]}$ photoluminescence (PL) quantum yields reaching near-unity, ${ }^{[4-6]}$ directed emission profile, ${ }^{[7-9]}$ switchable excitonic polarization, ${ }^{[10]}$ and spectral tunability as a result of their lateral size and thickness control at monolayer (ML) precision. ${ }^{[11-13]}$ These quasi-2D structures further enable unique opportunities for the design of novel heterostructures as a result of the possibilities of isotropically growing a shell around the entire core seed with atomic layer precision $^{[12,14,15]}$ and/or anisotropically only a crown $^{[16-18]}$ with an identical number of monolayers fixes vertical thickness as the seed core, as an purely lateral extension (wings) which is otherwise not possible at such a level of selectivity for other types of solution-processed nanocrystals in different geometries, for example quantum dots (QDs). Using heterostructures of NPLs, amplified spontaneous emission (ASE) with ultralow thresholds, ${ }^{[19-23]}$ large modal gain, ${ }^{[19,21]}$ long gain lifetime, ${ }^{[21,24]}$ and stable $\operatorname{ASE}^{[21,25]}$ and lasing ${ }^{[26]}$ have been recently reported in the green and red regions of the visible spectrum using $4 \mathrm{ML} \mathrm{CdSe} / \mathrm{CdS}$ core/crown, $\mathrm{CdSe} / \mathrm{Cd}_{1-x} \mathrm{Zn}_{x} \mathrm{~S}$ core/shell, and $\mathrm{CdSe} / \mathrm{CdS} / \mathrm{Cd}_{1-x} \mathrm{Zn}_{x} \mathrm{~S}$ core/crown/shell NPLs. However, these have not been achieved using hetero-NPLs in the blue region to date.

Since blue emission characteristically necessitates smaller nanocrystals which exhibit faster Auger rates and an increased density of trap surface traps states compared to larger ones in general and, therefore, achieving low threshold ASE and lasing in the blue region is unambiguously difficult for nanocrystals. ${ }^{[27]}$ However, it was demonstrated that NPLs display reduced Auger rates compared to QDs having a comparable bandgap as a result of the reduced exciton density due to the large lateral area of these 2D structures, ${ }^{[28-30]}$ which makes them suitable candidates for optical gain studies in the blue region. Yet, there are very few reports on optical gain of NPLs in the blue region compared to heavily studied green and red regions. This is largely due to the poor optical properties of 3 and $2 \mathrm{MLCdSe}$ NPLs that are in the form of 
(a)

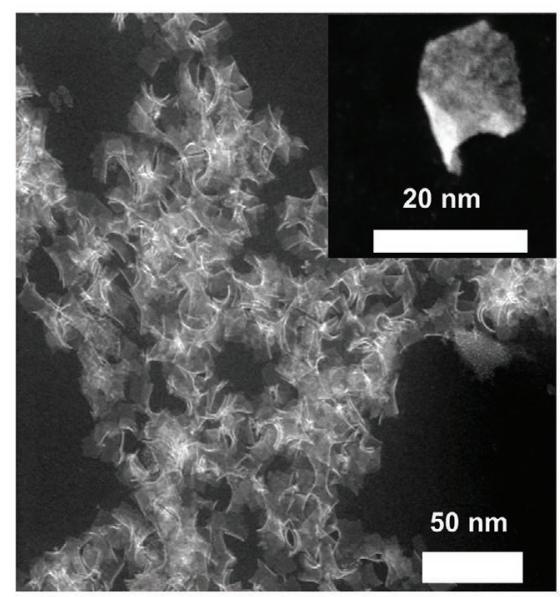

(b)

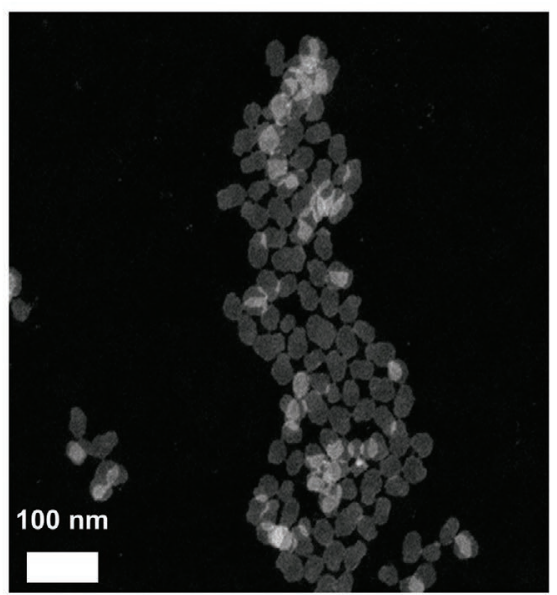

(c)

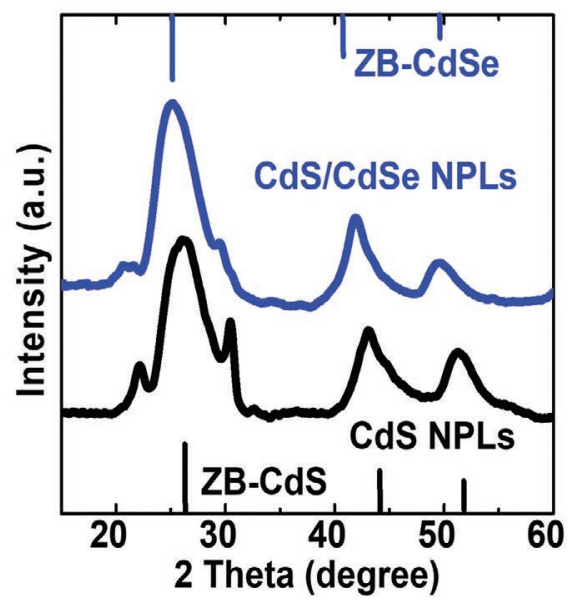

Figure 1. TEM images of a) $3 \mathrm{ML}$ CdS NPLs and b) $3 \mathrm{ML}$ CdS/CdSe core/crown NPLs (additionally coated with CdS shells). 3 ML CdS/CdSe core/ crown NPLs were coated with the CdS shell to better observe the shape and size since the as-synthesized 3 ML CdS/CdSe core/crown NPLs are rolled up as can be seen in Figure S2 of the Supporting Information. Inset of (a) shows a TEM image of an unrolled CdS core NPL. C) XRD spectra of 3 ML $\mathrm{CdS}$ and $\mathrm{CdS}-\mathrm{CdSe}$ NPLs.

only core having strong defect emission associated with the trapping of the carriers at the surface. Additionally, rolling of these NPLs ${ }^{[6]}$ makes uniform solid thin-film formation practically too difficult which is essential to reducing the optical losses in the ASE performance. To the best of our knowledge, there are solely two reports of optical gain in the blue region using NPLs, ${ }^{[30,31]}$ and the ASE thresholds of these previous works are between 50 and $250 \mu \mathrm{J} \mathrm{cm}^{-2}$, which are at least one order of magnitude larger than the ASE thresholds of NPLs in the green and red region. ${ }^{[2,32]}$ While these results are better than the finest reports from QDs, ${ }^{[3,34]}$ there is still a strong need for improvement toward their usage as gain media and to match the results in the green and red range. Among a variety of possible hetero-NPLs quantum rings (QRs) that can be conveniently obtained by growing a narrow bandgap crown around a wide bandgap core in NPLs have not been considered for optical gain in blue thus far. For example, even though inverted type-I CdS/CdSe core/crown NPLs have been shown to make QRs with continuously tunable emission in the blue region, the optical gain properties of these very interesting and intriguing 2D heterostructures have never been explored till date.

In this study, we show high performance blue ASE in its class with an ultralow threshold of $2.7 \mu \mathrm{J} \mathrm{cm}^{-2}(\approx 1.6$ exciton per NPL), accompanied by a large net modal gain coefficient of $360 \mathrm{~cm}^{-1}$, enabled by the engineered QRs in the form of $3 \mathrm{ML}$ CdS/CdSe core/crown NPLs. The ASE threshold and net modal gain of our QRs outperform the previously demonstrated ASE thresholds and net modal gain coefficients from the solutionprocessed nanoparticles of all types in the comparable spectral range. The large absorption cross-section and intrinsically slow Auger rates of our hetero-NPLs lead to such an ultralow level of ASE thresholds in this 2D system. As an important benefit, we also demonstrate that the ASE peak can be continuously spectrally adjusted via controlling the geometry of the resulting QR by modifying the lateral size of the crown.

\section{Results and Discussion}

We synthesized CdS/CdSe core/crown NPLs using 3 ML CdS NPLs as the starting cores. The details of the synthesis of 3 ML CdS NPLs were given in the Supporting Information. CdSe crowns in different lateral sizes were grown on the seed CdS NPLs at $170-175{ }^{\circ} \mathrm{C}$ after the injection of cadmium and selenium precursors at room temperature. The crown growth temperature is critically important to obtain QRs with uniform thickness. The growth at higher temperatures leads to emission around $\approx 510 \mathrm{~nm}$ together with additional emission around $\approx 460 \mathrm{~nm}$. This can be explained by the conversion of some of $3 \mathrm{ML}$ CdS cores to $4 \mathrm{ML}$ CdS cores at high temperatures as previously reported ${ }^{[35]}$ and the growth of $4 \mathrm{ML}$ CdSe crown on these 4 ML NPLs. Addition of a small amount of oleic acid (few hundred microliters) is also needed to suppress the transformation of these 3 ML CdS NPLs to 4 ML CdS NPLs during the growth of CdSe crown while the addition of a large amount of oleic acid hinders the growth of CdSe crown as a result of the formation of cadmium oleate in the presence of oleic acid together with cadmium acetate. The lateral size of the crown can be tuned by adjusting the amount of precursors needed for the crown growth. ${ }^{[11]}$ The transmission electron microscopy (TEM) images of seed CdS and CdS/CdSe NPLs having medium crown are given in Figure 1a,b. The obtained CdS/CdSe NPLs feature a larger lateral length $(27 \pm 6 \mathrm{~nm})$ compared to the CdS core NPLs (14 $\pm 4 \mathrm{~nm})$, as can be seen in TEM images, signifying the successful growth of CdSe crown around CdS core NPLs. X-ray diffraction (XRD) spectra of the synthesized CdS core and CdS/ CdSe core/crown NPLs are presented in Figure 1c. As can be seen from the diffraction pattern, CdS core and CdS/CdSe NPLs have a zinc-blend crystal structure. The broadening of the XRD peaks in these only 3 ML thick NPLs is associated with their very thin vertical size. The apparent slight shift of the XRD peaks of the seed CdS NPLs toward lower angles is due to the dilation of the lattice in these core NPLs compared to the bulk. ${ }^{[35]}$ 

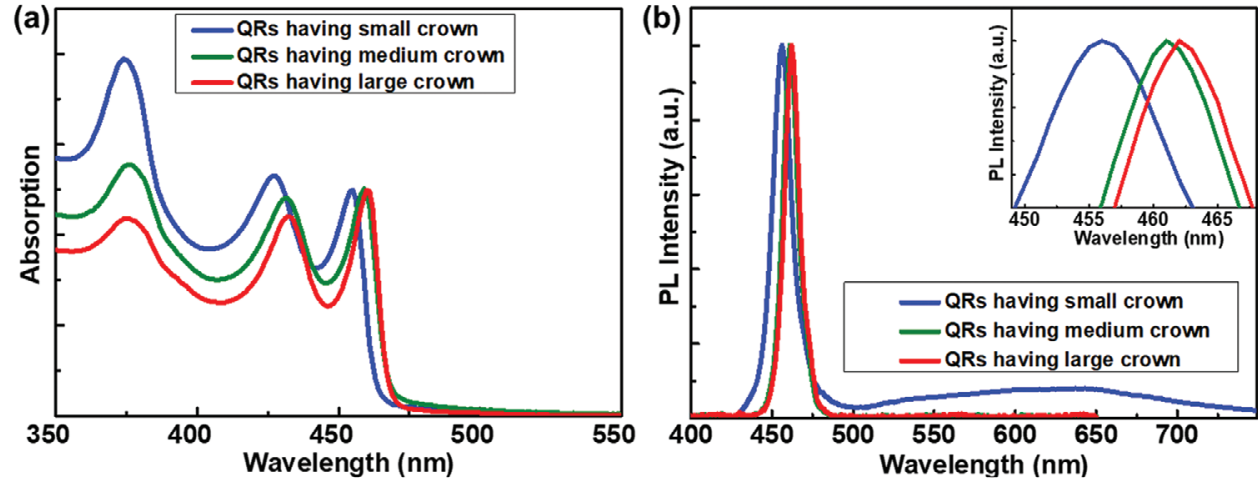

Figure 2. a) Absorption spectra of QRs having CdSe crown in different lateral sizes. The absorption spectra are normalized at the peak of heavy hole transition. b) PL spectra of QRs having CdSe crown in different lateral sizes. Inset of (b) shows zoom-in PL spectra of QRs at a narrower spectral window to show the PL peaks clearly.

As shown in Figure 1c, the XRD peaks are shifted slightly to the lower angles as CdSe crown forms around the periphery of core CdS NPLs. This shift is due to the larger lattice constant of CdSe, which induces strain with the growth of CdSe crown.

The absorption spectra of CdS/CdSe NPLs are shown in Figure 2a. The peak at $\approx 375 \mathrm{~nm}$ resolved in the absorption spectra of CdS/CdSe NPLs is associated with heavy and light hole transitions taking place in $\mathrm{CdS}^{[6]}$ as $3 \mathrm{ML}$ CdS core NPLS exhibit an excitonic transition at $\approx 375 \mathrm{~nm}$ as can be seen in Figure S1 of the Supporting Information. The peak tunable between 454 and $462 \mathrm{~nm}$ is related with heavy hole transition taking place in the CdSe crown in different lateral sizes. ${ }^{[2]}$ This is due to the lateral confinement effect felt by the generated excitons in the CdSe crown. ${ }^{[11]}$ The peak between 429 and $434 \mathrm{~nm}$ is due to the light hole transition in the CdSe crown. The absorption cross-section of these NPLs was also calculated by following our previous report. ${ }^{[36]}$ The absorption cross-sections of the NPLs at $400 \mathrm{~nm}$ having heavy hole transitions at $459.4 \mathrm{~nm}$ (QRs having medium crown) is $2.7 \times 10^{-13} \mathrm{~cm}^{2}$. The PL spectra of the synthesized NPLs in solution at room temperature are presented in Figure $2 \mathrm{~b}$. The PL peak of the NPLs is tunable from 456 to $462 \mathrm{~nm}$, as can be seen clearly in the inset of Figure $2 \mathrm{~b}$ owing to the controlled lateral confinement in the crown, which provides a tuning knob for determining the spectral window of the optical gain. In addition, QRs having small crown also exhibit broad emission centered at $\approx 640 \mathrm{~nm}$, which is associated with presence of trap states in these QRs as result of the nonuniform growth of crown around the seed CdS NPLs. ${ }^{[11]}$ The full-width-half-maximum (FWHM) of these NPLs drop from $14 \mathrm{~nm}$ for QRs having small crowns to $10 \mathrm{~nm}$ for QRs having medium and large crowns. The relatively larger PL FWHM of inverted NPLs having smaller crowns is a result of the variation in lateral confinement as shown in QRs of NPLS previously. ${ }^{[11]}$ In addition, the existence of trap emission generally observed in $3 \mathrm{ML}$ CdSe NPLs ${ }^{[4,15]}$ is largely passivated in these QRs except for QRs having thin CdSe crown as can be observed in Figure 2b.

To investigate the ASE performance of our QRs, we prepared solid films on the precleaned quartz substrates by spin-coating our QRs dispersed in hexane $\left(\approx 30 \mathrm{mg} \mathrm{mL}^{-1}\right)$ and the obtained films were excited in a stripe geometry (using a cylindrical lens) at $400 \mathrm{~nm}$ using mode-locked femtosecond pulses with a $120 \mathrm{fs}$ pulse width and at a $1 \mathrm{kHz}$ repetition rate. ASE was characterized by collecting the emitted photons along the stripe using a spectrometer coupled to a fiber. Figure 3a displays pumpfluence dependence of ASE spectra of the films of CdS/CdSe (a)

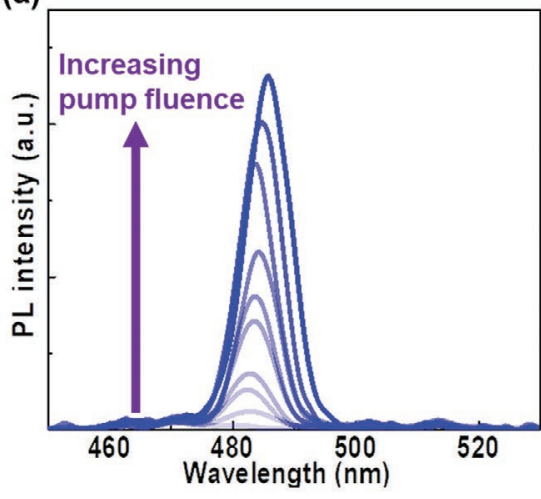

(b)

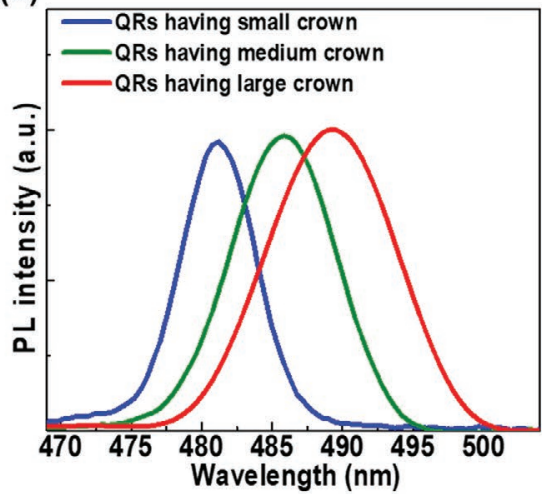

(c)

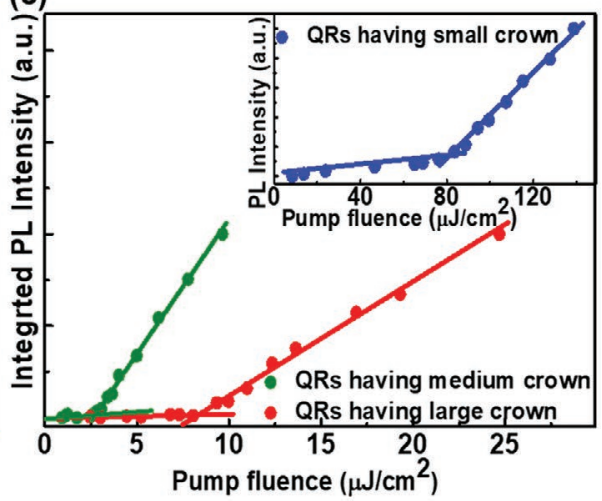

Figure 3. a) Pump fluence dependence of PL spectra of QRs having medium CdSe crown. ASE emerges with the increase of pump fluence. b) ASE spectra of QRs having CdSe crown in different lateral sizes. The ASE peak moves from 481 to $492 \mathrm{~nm}$ as the lateral size of the QRs is increased. c) Integrated PL intensity of QRs having medium and large crowns. The inset of (c) shows integrated PL intensity of QRs having thin crowns. The ASE threshold of QRs having thin crowns is significantly higher than the ASE threshold of QRs having medium and large crowns. 

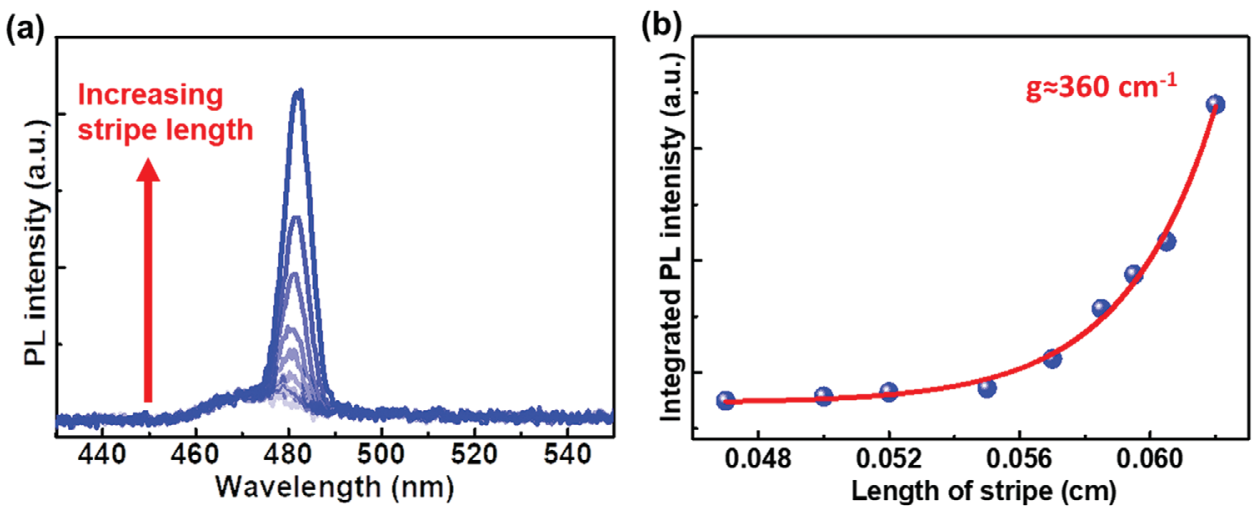

Figure 4. a) ASE measurements at different stripe lengths and b) the resulting integrated PL intensity as a function of the stripe length at a pump fluence of $92 \mu \mathrm{cm}^{-2}$ for QRs having medium crown. The fitting of the variable stripe length measurements (red curve) results in a net gain coefficient of $360 \mathrm{~cm}^{-1}$.

NPLs with medium crown as an exemplary case. The pumpfluence dependence of the ASE spectra from rest of the QR films are shown in Figures S3 and S4 of the Supporting Information. The spontaneous emission peak can be observed at lower pump fluences at $\approx 480 \mathrm{~nm}$ with an FWHM of $\approx 18 \mathrm{~nm}$ (which can be better observed in Figure 4a), while the sharp ASE peak with an FWHM of $8 \mathrm{~nm}$ appears at higher fluences on the red side of the spontaneous emission. The spontaneous emission from the solid films of these QRs having medium crown shows almost purely bandedge emission unlike the films of 3 ML CdSe NPLs showing very strong defect emission, which can be better seen in Figure $2 b$. This is likely due to the better passivation of the trap sites present on the surface of our ultrathin QRs. The ASE peak is redshifted by $\approx 5 \mathrm{~nm}$ relative to the spontaneous emission peak as can be seen easily in Figure 4a. The red shift of ASE indicates multiexcitonic gain, which is commonly observed in type-I NPLs and QDs. This red shifted ASE is beneficial for optical gain since this leads to a reduction in the self-absorption. ${ }^{[21,34]}$ The ASE spectra of our QRs having different crown sizes are presented in Figure $3 \mathrm{~b}$. The ASE peaks cover a range of $10 \mathrm{~nm}$ from $\approx 480$ to $\approx 490 \mathrm{~nm}$. The confinement control in the lateral size of our QRs provides a tuning knob for continuously adjusting the spectrum of ASE and a new route for fine tuning the optical gain spectra in these NPLs having discrete spectrum.

Total emission intensities as a function of the pump fluence for all the investigated samples are shown in Figure 3c. The threshold varies as we change the size of the crown as can be observed in Figure 3c. The lowest achieved threshold is $2.7 \mu \mathrm{J} \mathrm{cm}^{-2}$ from the sample with medium crown. At this threshold, $\approx 1.6$ excitons per QR were generated by the pump laser. The previously best reported threshold in the blue range is $\approx 8 \mu \mathrm{J} \mathrm{cm}^{-2}$ from perovskite nanocrystals. ${ }^{[37]}$ The QRs with large crown exhibit ASE threshold of $\approx 8.3 \mu \mathrm{J} \mathrm{cm}^{-2}$. This difference in the thresholds between these two samples can be attributed to the difference in film formation and surface passivation and variation in film thickness, which altogether strongly affect the optical confinement factor and optical losses. However, the sample with the small crown has a distinctly higher threshold $\left(\approx 81 \mu \mathrm{J} \mathrm{cm}^{-2}\right)$. This can be explained by the stronger Auger rate as a result of higher exciton density and smaller absorption cross-section of this sample. Also, there could be a possible contribution of the presence of trap sites, which lead to strong deep trap emission in the films of these QRs having thin crowns, to this higher threshold. However, the stimulated emission kinetically outcompetes this emission from trap sites above the threshold as the ASE peak appears above the threshold in this sample having strong trap emission as presented in Figure S3 of the Supporting Information. As demonstrated in previous works of NPLs, the stimulated emission rate is an order magnitude faster than the hole trapping. ${ }^{[6,30,38]}$ This suggests that trap sites do not significantly affect the ASE threshold since the stimulated emission is significantly faster than the trapping of the photoexcited carriers.

The modal gain of these films of our QRs was obtained employing the variable-stripe-length (VSL) method. ${ }^{[39]}$ Figure $4 a$ presents the emission spectra of VSL measurements (at a pump fluence of $92 \mu \mathrm{J} \mathrm{cm}^{-2}$ ) of the QRs having a medium size crown that show the best ASE performance. Figure $4 \mathrm{~b}$ shows the total emission intensity as a function of the stripe length and the gain coefficient obtained from the fitting is $360 \mathrm{~cm}^{-1}$. In here, we employed a fitting function of $I=\frac{A}{g}\left(e^{g L}-1\right),{ }^{[39]}$ where $A$ is a parameter that reflects spontaneous emission intensity, $g$ is the net modal gain coefficient, and $L$ is the length of the excitation stripe to obtain net modal gain coefficient. This obtained gain coefficient is fourfold larger than the previously best reported gain coefficient of solution processed materials ${ }^{[31]}$ in the similar spectral window. This can be attributed to the large intrinsic absorption coefficient of $3 \mathrm{ML}$ CdS/CdSe core/crown NPLs, which makes them excellent candidates as optical gain media as the intrinsic absorption shows the gain capability of a material. ${ }^{[13,40,41]}$ The previously reported best gain coefficient in the blue region for solution-processed materials is also from core 3 ML CdSe NPLs demonstrating the potential of these thin QRs of CdS/CdSe NPLs. ${ }^{[31]}$

\section{Conclusion}

In summary, synthesizing QRs engineered from $3 \mathrm{ML}$ CdS/CdSe core/crown NPLs, we demonstrated high-performance ASE 
with a record low threshold of $\approx 2.7 \mu \mathrm{J} \mathrm{cm}^{-2}$ together with a large net modal gain coefficient of $360 \mathrm{~cm}^{-1}$ in the challenging blue region for solution processed materials. We also showed that their ASE peak can be conveniently spectrally tuned by controlling the lateral confinement in the $\mathrm{QR}$ architecture provided by the crown. The resulting ASE threshold and net modal gain of our QRs surpass the ASE thresholds and net modal gain coefficients of the state-of-the-art solution processed materials in the similar spectral window. The obtained gain coefficient is fourfold larger than the previously reported best gain coefficient of solution-processed materials in the similar spectral range. The low ASE threshold of our QRs stems from the large absorption cross-section, negligible dispersion both in thickness and in lateral size as indicated by narrow PL linewidth and intrinsically slow Auger rates compared to QDs. The achieved record low ASE threshold is an important step toward realizing continuouswave pumped lasing in the blue region. Thus, these findings indicate that our engineered QRs make an exceptional candidate as the colloidal gain media for lasers in the blue region with their ultralow threshold ASE and large net modal gain.

\section{Supporting Information}

Supporting Information is available from the Wiley Online Library or from the author.

\section{Acknowledgements}

The authors gratefully acknowledge the financial support in part from Singapore National Research Foundation under the programs of NRFNRFI2016-08 and NRF-CRP14-2014-03 and the Science and Engineering Research Council, Agency for Science, Technology and Research (A*STAR) of Singapore and in part from TUBITAK 115E679. H.V.D. also acknowledges support from TUBA.

\section{Conflict of Interest}

The authors declare no conflict of interest.

\section{Data Availability Statement}

Data available on request from the authors.

\section{Keywords}

amplified spontaneous emission, blue optical gain, colloidal quantum wells, nanoplatelet heterostructures, quantum rings

Received: January 17, 2021

Published online: February 15, 2021

[1] J. Zhang, Y. Sun, S. Ye, J. Song, J. Qu, Chem. Mater. 2020, 32, 9490.

[2] S. Ithurria, M. D. Tessier, B. Mahler, R. P. S. M. Lobo, B. Dubertret, A. L. Efros, Nat. Mater. 2011, 10, 936.

[3] S. Ithurria, B. Dubertret, J. Am. Chem. Soc. 2008, 130, 16504.
[4] M. Sharma, K. Gungor, A. Yeltik, M. Olutas, B. Guzelturk, Y. Kelestemur, T. Erdem, S. Delikanli, J. R. McBride, H. V. Demir, Adv. Mater. 2017, 29, 1700821.

[5] B. Liu, Y. Altintas, L. Wang, S. Shendre, M. Sharma, H. Sun, E. Mutlugun, H. V. Demir, Adv. Mater. 2020, 32, 2070054.

[6] S. Delikanli, G. Yu, A. Yeltik, S. Bose, T. Erdem, J. Yu, O. Erdem, M. Sharma, V. K. Sharma, U. Quliyeva, S. Shendre, C. Dang, D. H. Zhang, T. C. Sum, W. Fan, H. V. Demir, Adv. Funct. Mater. 2019, 29, 1901028.

[7] R. Scott, J. Heckmann, A. V. Prudnikau, A. Antanovich, A. Mikhailov, N. Owschimikow, M. Artemyev, J. I. Climente, U. Woggon, N. B. Grosse, A. W. Achtstein, Nat. Nanotechnol. 2017, 12, 1155.

[8] Y. Gao, M. C. Weidman, W. A. Tisdale, Nano Lett. 2017, 17, 3837.

[9] S. Shendre, S. Delikanli, M. Li, D. Dede, Z. Pan, S. T. Ha, Y. H. Fu, P. L. Hernández-Martínez, J. Yu, O. Erdem, A. I. Kuznetsov, C. Dang, T. C. Sum, H. V. Demir, Nanoscale 2019, 11, 301.

[10] A. Najafi, S. Tarasek, S. Delikanli, P. Zhang, T. Norden, S. Shendre, M. Sharma, A. Bhattacharya, N. Taghipour, J. Pientka, H. V. Demir, A. Petrou, T. Thomay, ACS Appl. Nano Mater. 2020, 3, 3151.

[11] S. Delikanli, B. Guzelturk, P. L. Hernández-Martínez, T. Erdem, Y. Kelestemur, M. Olutas, M. Z. Akgul, H. V. Demir, Adv. Funct. Mater. 2015, 25, 4282.

[12] S. Ithurria, D. V. Talapin, J. Am. Chem. Soc. 2012, 134, 18585.

[13] A. Di Giacomo, C. Rodà, A. H. Khan, I. Moreels, Chem. Mater. 2020, 32, 9260.

[14] F. Muckel, S. Delikanli, P. L. Hernández-Martínez, T. Priesner, S. Lorenz, J. Ackermann, M. Sharma, H. V. Demir, G. Bacher, Nano Lett. 2018, 18, 2047.

[15] S. Delikanli, M. Z. Akgul, J. R. Murphy, B. Barman, Y. Tsai, T. Scrace, P. Zhang, B. Bozok, P. L. Hernández-Martínez, J. Christodoulides, A. N. Cartwright, A. Petrou, H. V. Demir, ACS Nano 2015, 9, 12473.

[16] M. D. Tessier, P. Spinicelli, D. Dupont, G. Patriarche, S. Ithurria, B. Dubertret, Nano Lett. 2014, 14, 207.

[17] Y. Kelestemur, M. Olutas, S. Delikanli, B. Guzelturk, M. Z. Akgul, H. V. Demir, J. Phys. Chem. C 2015, 119, 2177.

[18] M. Sharma, S. Delikanli, H. V. Demir, Proc. IEEE 2020, 108, 655.

[19] B. Guzelturk, Y. Kelestemur, M. Olutas, S. Delikanli, H. V. Demir, ACS Nano 2014, 8, 6599.

[20] C. She, I. Fedin, D. S. Dolzhnikov, A. Demortière, R. D. Schaller, M. Pelton, D. V. Talapin, Nano Lett. 2014, 14, 2772.

[21] N. Taghipour, S. Delikanli, S. Shendre, M. Sak, M. Li, F. Isik, I. Tanriover, B. Guzelturk, T. C. Sum, H. V. Demir, Nat. Commun. 2020, 11, 3305.

[22] D. Dede, N. Taghipour, U. Quliyeva, M. Sak, Y. Kelestemur, K. Gungor, H. V. Demir, Chem. Mater. 2019, 31, 1818.

[23] L. Zhang, H. Yang, B. Yu, Y. Tang, C. Zhang, X. Wang, M. Xiao, Y. Cui, J. Zhang, Adv. Opt. Mater. 2020, 8, 1901615.

[24] B. Guzelturk, Y. Kelestemur, M. Olutas, Q. Li, T. Lian, H. V. Demir, J. Phys. Chem. Lett. 2017, 8, 5317.

[25] Y. Kelestemur, B. Guzelturk, O. Erdem, M. Olutas, K. Gungor, H. V. Demir, Adv. Funct. Mater. 2016, 26, 3570.

[26] M. Sak, N. Taghipour, S. Delikanli, S. Shendre, I. Tanriover, S. Foroutan, Y. Gao, J. Yu, Z. Yanyan, S. Yoo, C. Dang, H. V. Demir, Adv. Funct. Mater. 2020, 30, 1907417.

[27] V. I. Klimov, A. A. Mikhailovsky, D. W. McBranch, C. A. Leatherdale, M. G. Bawendi, Science 2000, 287, 1011.

[28] E. Baghani, S. K. O'Leary, I. Fedin, D. V. Talapin, M. Pelton, J. Phys. Chem. Lett. 2015, 6, 1032.

[29] L. T. Kunneman, M. D. Tessier, H. Heuclin, B. Dubertret, Y. V. Aulin, F. C. Grozema, J. M. Schins, L. D. A. Siebbeles, J. Phys. Chem. Lett. 2013, 4, 3574.

[30] B. T. Diroll, D. V. Talapin, R. D. Schaller, ACS Photonics 2017, 4, 576.

[31] C. She, I. Fedin, D. S. Dolzhnikov, P. D. Dahlberg, G. S. Engel, R. D. Schaller, D. V. Talapin, ACS Nano 2015, 9, 9475. 
[32] J. Q. Grim, S. Christodoulou, F. Di Stasio, R. Krahne, R. Cingolani, L. Manna, I. Moreels, Nat. Nanotechnol. 2014, 9, 891.

[33] B. Guzelturk, Y. Kelestemur, M. Z. Akgul, V. K. Sharma, H. V. Demir, J. Phys. Chem. Lett. 2014, 5, 2214.

[34] C. Dang, J. Lee, C. Breen, J. S. Steckel, S. Coe-Sullivan, A. Nurmikko, Nat. Nanotechnol. 2012, 7, 335.

[35] Z. Li, H. Qin, D. Guzun, M. Benamara, G. Salamo, X. Peng, Nano Res. 2012, 5, 337.

[36] A. Yeltik, S. Delikanli, M. Olutas, Y. Kelestemur, B. Guzelturk, H. V. Demir, J. Phys. Chem. C 2015, 119, 26768.
[37] S. Yakunin, L. Protesescu, F. Krieg, M. I. Bodnarchuk, G. Nedelcu, M. Humer, G. De Luca, M. Fiebig, W. Heiss, M. V. Kovalenko, Nat. Commun. 2015, 6, 8056.

[38] L. T. Kunneman, J. M. Schins, S. Pedetti, H. Heuclin, F. C. Grozema, A. J. Houtepen, B. Dubertret, L. D. A. Siebbeles, Nano Lett. 2014 14, 7039.

[39] K. L. Shaklee, R. E. Nahory, R. F. Leheny, J. Lumin. 1973, 7, 284.

[40] A. W. Achtstein, A. Antanovich, A. Prudnikau, R. Scott, U. Woggon, M. Artemyev, J. Phys. Chem. C 2015, 119, 20156.

[41] S. Bisschop, P. Geiregat, T. Aubert, Z. Hens, ACS Nano 2018, 12, 9011. 